\title{
Fatigue Assessment Model of Corroded RC Beams Strengthened with Prestressed CFRP Sheets
}

\author{
Li Song ${ }^{1,2)}$, and Jian $\mathrm{Hou}^{3), *}$
}

(Received March 10, 2016, Accepted January 31, 2017, Published online May 19, 2017)

\begin{abstract}
This paper presents a fatigue assessment model that was developed for corroded reinforced concrete (RC) beams strengthened using prestressed carbon fiber-reinforced polymer (CFRP) sheets. The proposed model considers the fatigue properties of the constituent materials as well as the section equilibrium. The model provides a rational approach that can be used to explicitly assess the failure mode, fatigue life, fatigue strength, stiffness, and post-fatigue ultimate capacity of corroded beams strengthened with prestressed CFRP. A parametric analysis demonstrated that the controlling factor for the fatigue behavior of the beams is the fatigue behavior of the corroded steel bars. Strengthening with one layer of non-prestressed CFRP sheets restored the fatigue behavior of beams with rebar at a low corrosion degree to the level of the uncorroded beams, while strengthening with 20and 30\%-prestressed CFRP sheets restored the fatigue behavior of the beams with medium and high corrosion degrees, respectively, to the values of the uncorroded beams. Under cyclic fatigue loading, the factors for the strengthening design of corroded RC beams fall in the order of stiffness, fatigue life, fatigue strength, and ultimate capacity.
\end{abstract}

Keywords: fatigue assessment model, corrosion, reinforced concrete beam, prestressed CFRP sheets.

\section{Introduction}

Infrastructure such as bridges and marine structures are prone to corrosion, and these structures are usually subjected to repeated loading. Corrosion reduces the steel area, worsens the steel properties, weakens the bond between the concrete and steel bars, and results in cracking and spalling of the concrete cover (Bigaud and Ali 2014; Almusallam 2001). Fatigue is a process of progressive and internal structural changes in a material that is subjected to repetitive stresses. For example, the primary girders of bridges are subjected to many traffic loading cycles every day, which can lead to failure due to these repetitive tensile stresses. Although corrosion and fatigue processes have been extensively studied, the coupled effect of these two processes has rarely been studied (Bastidas-Arteaga et al. 2009; Ai-Hammoud et al. 2011; Yi et al. 2010). Several experimental studies have shown that localized corrosion leading to pitting may provide sites for fatigue crack initiation and that corrosive agents increase the fatigue crack growth rate (Bastidas-Arteaga et al. 2009; Ai-Hammoud et al. 2010).

\footnotetext{
${ }^{1)}$ School of Civil Engineering, Central South University, 22 Shaoshan South Road, Changsha, China.

${ }^{2)}$ National Engineering Laboratory for High Speed Railway Construction, Changsha, China.

${ }^{3)}$ Department of Civil Engineering, Xi'an Jiaotong University, Xi'an, China.

*Corresponding Author; E-mail: houjian0323@126.com Copyright (c) The Author(s) 2017. This article is an open access publication
}

The interactive effect of corrosion and fatigue is more damaging than the sum of the damage caused by the components individually. The loss of strength and stiffness can thus be exaggerated if corrosion is combined with fatigue loading (Bastidas-Arteaga et al. 2009; Masoud et al. 2001).

Structural engineers face the challenge of assessing the vulnerability of deteriorated structures and deciding on appropriate strengthening methods. Externally bonded carbon fiber-reinforced polymer (CFRP) sheets have been widely used to restore or increase the capacities of reinforced concrete (RC) beams due to their advantages including their low weight, high strength and stiffness, high durability, and ease of application (Kang et al. 2012; Ouezdou et al. 2009; Grelle and Sneed 2013; Ren et al. 2015). Many studies have been conducted to study the effect of CFRP sheets on the performance of RC beams (Al-Rousan and Issa 2011; Oudah and El-Hacha 2013a, b; EISafty et al. 2014; Kang et al. 2014). However, several researchers have demonstrated that applying CFRP sheets as externally bonded strips for flexural strengthening of RC structural elements uses only $20-30 \%$ of their strength (Motavalli et al. 2011). Additionally, the serviceability of RC beams is not generally affected by CFRP sheet repair due to the relatively small increase in stiffness provided by CFRP sheets. The strips are better used when the CFRP material is prestressed. Similar to the advantages of conventional prestressed concrete compared to $\mathrm{RC}$, prestressed, externally bonded reinforcement has several advantages over externally bonded reinforcement including reduced crack widths, reduced deflections, reduced stress in the internal steel, and increased fatigue resistance (Michels et al. 2013; Correia et al. 2015). Triantafillou et al. 
(1992) tested beams strengthened with prestressed CFRP sheets and found that prestressed CFRP sheets significantly contributed to the improved cracking and deflection of the strengthened beams. El-Hacha et al. (2001) provided a general summary of the prestressed CFRP sheet application, including feasible prestressing techniques for concrete structures. Kim et al. (2008) tested prestressed RC beams that had been strengthened with prestressed CFRP sheets and found that using prestressed CFRP sheets resulted in less localized damage and that the level of prestress in the sheets significantly contributed to the ductility and cracking behavior of the strengthened beams. Wang et al. (2012) investigated the flexural behavior and long-term prestress losses of RC beams strengthened with post-tensioned CFRP sheets and noted that the prestress losses of CFRP sheets in the post-tension system could be primarily attributed to the anchorage set, while the time-dependent losses caused by creep, concrete shrinkage and relaxation of the CFRP sheets were relatively small. Rosenboom and Rizkalla (2006) investigated the fatigue behavior of prestressed concrete bridge girders strengthened with various CFRP systems using various strengthening levels, prestressing configurations, and fatigue loading ranges. The test results showed that CFRP strengthening could reduce crack width, crack spacing, and the induced stress ratio in the prestressed strands under service loading conditions. Although a sufficient number of studies have investigated the strength, serviceability, prestress losses, and CFRP systems in general, few studies have investigated the fatigue performance of members with prestressed CFRP sheet applications. The fatigue performance of corroded RC beams strengthened with prestressed CFRP sheets has not yet been discussed.

Additionally, the development of a fatigue assessment model (FAM) for corroded members is not an easy task due to the many relevant parameters and variables that determine the fatigue behavior. Several attempts have been made to develop an FAM for corroded beams. The most common approaches are the stress-life and strain-life models (Ma et al. 2014; Elrefai et al. 2012). These models provide the fatigue life of members but do not predict the strain and stress distributions across the beam. Song and Yu (2015) tested beams with corroded steel reinforcement (corroded beams) strengthened with CFRP sheets and proposed an analytical fatigue prediction model (FPM) to assess the fatigue behavior of the CFRP-strengthened corroded beams. In this study, the effect of prestressed CFRP systems on the fatigue behavior of corroded RC beams was not studied. None of the available FAMs are capable of accurately assessing the fatigue behavior of corroded beams strengthened with prestressed CFRP. This paper presents the second phase of a research project conducted to quantify the effect of CFRP systems on the fatigue behavior of corroded RC beams. The first phase of the project (Song and Yu 2015) aimed to examine the effect of steel corrosion and the nonprestressed CFRP systems on the fatigue behavior of corroded RC beams. The results showed that non-prestressed CFRP systems are applicable to RC beams with only low corrosion degrees $(0-4.6 \%)$. The second phase intends to develop an overall assessment model capable of assessing the fatigue behavior of corroded RC beams strengthened with prestressed CFRP sheets and to discuss whether prestressed CFRP systems are applicable to RC beams with medium and high corrosion degrees.

In this study, an FAM was developed to consider the fatigue behavior of corroded beams with prestressed CFRP sheets. The model provided a rational approach that can be used to explicitly assess the failure mode, fatigue life, fatigue strength, stiffness, and post-fatigue ultimate capacity of CFRP-strengthened corroded beams under fatigue loading by considering the influences of different corrosion degrees, load ranges, and prestressed levels.

\section{Fatigue Assessment Model}

An FAM based on the fatigue properties of the constituent materials and cross-sectional stress analysis was proposed to assess the fatigue behavior of corroded beams strengthened with prestressed CFRP sheets. The fatigue properties of the constituent materials of the CFRP-strengthened corroded beams are discussed first, followed by a study of the fatigue bond properties and the cross-sectional stress under cyclic loading; finally, the step-by-step procedure used to implement the developed model is presented.

\subsection{Fatigue Properties of the Constituent Materials}

To assess the fatigue behavior of a composite member, the effects of cyclic loading on each constituent component must first be understood. If any of the constituent components reaches its fatigue limit prior to the required life of the member, the member will not be capable of carrying the required loads. These loading cycles contribute to the continuous deterioration of the constitutive materials. The models of the fatigue deterioration of concrete, corroded steel bars, and CFRP sheets are detailed in the following paragraphs.

\subsubsection{Concrete}

Bridge girders are one of the most common structural components that are subjected to cyclic loading. The beam test results showed that the concrete softened for all of the applied compression stress ranges, resulting in a decrease in the concrete elastic modulus and a corresponding increase in the concrete strain (Hefferan et al. 2004). Therefore, it is necessary to examine the effect of fatigue loading on the strength and strain of concrete to accurately determine the fatigue behavior of CFRP-strengthened RC beams (Oudah and El-Hacha 2013a, b). To evaluate the concrete fatigue damage, Holmen (1982) proposed that the total $\varepsilon_{\mathrm{c}}$ is the sum of two components: the first component is related to the endurance of the specimen $\varepsilon_{\mathrm{ce}}$, and the second component is the strain increment or plastic strain $\varepsilon_{\mathrm{cr}}$ related to the loading conditions and the number of cycles. The strain increment of concrete describes the unrecoverable component of concrete deformation. After $n$ cycles between the minimum and 
maximum stresses of $\sigma_{\mathrm{c}, \min }$ and $\sigma_{\mathrm{c}, \max }$, the plastic strain is expressed as follows (Song and Yu 2015):

$$
\varepsilon_{\mathrm{cr}}=\frac{f_{\mathrm{c}}}{E_{\mathrm{c}}} n^{0.29} \log ^{-1}\left(3.92 \alpha_{\mathrm{r}}-4.66\right)
$$

where $f_{\mathrm{c}}$ is the concrete compressive strength, $E_{\mathrm{c}}$ is the initial Young's modulus of the concrete, $n$ is the number of loading cycles, and $\alpha_{\mathrm{r}}$ is the stress ratio coefficient, which is expressed as follows:

$$
\alpha_{\mathrm{r}}=\frac{\sigma_{\mathrm{c}, \text { max }}-\sigma_{\mathrm{c}, \text { min }}}{f_{\mathrm{c}}-\sigma_{\mathrm{c}, \text { min }}}
$$

The Young's modulus of the concrete under repeated loading can be written as:

$$
E_{c}^{f}=\frac{\sigma_{c, \max }}{\left(\varepsilon_{c e}-\varepsilon_{c 0}\right)+\varepsilon_{c r}}
$$

where $\varepsilon_{\mathrm{ce}}$ is the strain under sustained loading, which is equal to $\sigma_{\mathrm{c}, \max } / E_{\mathrm{c}}$, and $\varepsilon_{\mathrm{c} 0}$ is the initial tensile strain of the concrete related to the level of prestress in the CFRP sheets.

Then, the evolution of the Young's modulus induced by fatigue loading can be derived from Eqs. (1) and (3):

$$
E_{\mathrm{c}}^{\mathrm{f}}=\sigma_{\mathrm{c}, \max } /\left\{\frac{\sigma_{\mathrm{c}, \max }}{E_{\mathrm{c}}}-\varepsilon_{\mathrm{c} 0}+\frac{f_{\mathrm{c}}}{E_{\mathrm{c}}} \times n^{0.29} \lg ^{-1}\left(3.92 \alpha_{\mathrm{r}}-4.66\right)\right\}
$$

Fatigue is a process of progressive internal damage in a material that has been subjected to repeated loading and is attributed to the propagation of internal micro-cracks; fatigue typically results in a significant increase in unrecoverable strain. The test results showed that fatigue failure of concrete is likely to occur when the plastic strain $\varepsilon_{\text {cr }}$ reaches a threshold criterion. The fatigue-failure criterion of concrete can be estimated as follows (Song and Yu 2015):

$$
\varepsilon_{\mathrm{cr}} \geq 0.4 f_{\mathrm{c}} / E_{\mathrm{c}}
$$

\subsubsection{Corroded Steel Bars}

The corrosion of steel reinforcement has commonly been associated with both the carbonation of concrete and chloride ingress. The former causes a more uniform attack with a relatively limited reduction of the rebar cross-sectional area, while chloride may cause severe pitting in the rebar with highly localized reductions in the cross-sectional area; therefore, corrosion due to chloride ions is discussed in this section. Pitting corrosion decreases the steel cross-sectional area via small pit nucleation. These pits propagate over time due to corrosion, and localized corrosion that leads to pitting may provide sites for fatigue crack initiation. Experimental studies have shown that pitting corrosion is responsible for the nucleation of fatigue cracks. In this case, corrosion pits tend to increase the formation of fatigue-crack nucleation points and crack growth (Bastidas-Arteaga et al. 2009). Fatigue-crack evolution further reduces the cross-sectional area of steel rebar, thus increasing steel stress. The reduced cross-sectional area becomes critical when the actual stress in the steel exceeds the yield strength of the steel, thus causing fatigue failure. Therefore, the reduced cross-sectional area of steel rebar can effectively denote the evolution of fatigue damage. The combined action of corrosion and fatigue loading significantly degrades the strength and deformability of rebar and decreases the ratio between the yield strength and ultimate strength of the rebar. To ensure the safety of a corroded beam, the steel stress is assumed to be equal to the yield strength when rebar fatigue failure occurs. Because the fatigue load range is constant throughout the fatigue loading process, the residual cross-sectional area of the corroded steel bar can be calculated as follows:

$$
A_{\mathrm{s}}^{\mathrm{f}}(N)=\sigma_{\mathrm{s}, \max } \cdot A_{\mathrm{sc}} / f_{\mathrm{yc}}
$$

where $A_{\mathrm{s}}^{\mathrm{f}}(N)$ is the residual cross-sectional area of the corroded steel subjected to $N$ cycles of repeated loading, $\sigma_{\text {s,max }}$ is the nominal maximum stress applied to the steel, $A_{\mathrm{s}}$ is the initial cross-sectional area of the steel, $A_{\mathrm{sc}}$ is the cross-sectional area of the corroded steel bars, and $f_{\mathrm{yc}}$ is the yield strength of the corroded steel.

The evolution of fatigue cracks in metal can generally be subdivided into three stages: fatigue crack nucleation, crack growth, and instability. The second stage is dominant and is characterized by a constant crack growth rate. As mentioned previously, corrosion pitting increases fatigue crack nucleation and provides sites for fatigue crack initiation, which is the cause of the fatigue crack propagation of corroded steel bars in the second stage. The fatigue damage area is assumed to follow a linear relationship with the ratio of the number of cycles to the fatigue life $n / N$; then, the fatigue damage area under every loading cycle is equal to $\left[A_{\mathrm{sc}}-A_{\mathrm{s}}^{\mathrm{f}}(N)\right] / N$. The residual area of the corroded steel $A_{\mathrm{s}}^{\mathrm{f}}(n)$ after being subjected to $n$ cycles of repeated loading can be calculated as:

$$
\begin{aligned}
A_{\mathrm{s}}^{\mathrm{f}}(n) & =A_{\mathrm{sc}}-\frac{n}{N}\left[A_{\mathrm{sc}}-A_{\mathrm{s}}^{\mathrm{f}}(N)\right] \\
& =A_{\mathrm{sc}}\left[1-(n / N) \cdot\left(1-\sigma_{\mathrm{s}, \max } / f_{\mathrm{yc}}\right)\right]
\end{aligned}
$$

Corrosion not only affects the steel area but also alters the steel's mechanical properties over time. The yield strength of corroded rebar can be expressed in terms of the strength of uncorroded rebar and the corrosion degree as follows (Song and Yu 2015):

$$
f_{\mathrm{yc}}=\alpha_{\mathrm{c}} \frac{A_{\mathrm{s} 0}}{A_{\mathrm{sc}}} f_{\mathrm{y} 0}=\frac{\alpha_{\mathrm{c}}}{1-\eta_{\mathrm{s}}} f_{\mathrm{y} 0}
$$

where $f_{\mathrm{y} 0}$ is the yield strength of the uncorroded steel bars, $A_{\mathrm{s} 0}$ is the cross-sectional area of the uncorroded rebar, $\alpha_{\mathrm{c}}$ is an empirical coefficient, and $\eta_{\mathrm{s}}$ is the average degree of corrosion of the rebar. Based on experimental data, the coefficient $\alpha_{\mathrm{c}}$ is considered to be equal to $1-1.196 \eta_{\mathrm{s}}$ (Song and $\mathrm{Yu}$ 2015).

The fatigue life $N$ is commonly determined using the stress-life $S-N$ method. In Eq. (7), the fatigue life $N$ is evaluated based on the constant stress amplitude fatigue test 
of steel samples at $\rho=0.1$, where $\rho=\sigma_{\mathrm{s}, \min } / \sigma_{\mathrm{s}, \max }$ (Song and $\mathrm{Yu}$ 2015):

$$
\lg N=\left(24.427+3.4 \eta_{\mathrm{s}}\right)-\left(7.6597+2.1 \eta_{\mathrm{s}}\right) \lg \Delta \sigma
$$

where $\Delta \sigma=\left(\sigma_{\max }-\sigma_{\min }\right)$ is the average nominal stress range and is determined by the first cycle.

Considering the test results in the literature, new studies must consider the effects of other fatigue variables, including the $\rho$ ratio, the stress concentration, and the pre-compressive stress, on the fatigue life of corroded steel. The stress-life $S-N$ curve of corroded steel rebar in RC beams can be modified as follows:

$$
\begin{aligned}
\lg N= & \left(24.427+3.4 \eta_{\mathrm{s}}\right)-\left(7.6597+2.1 \eta_{\mathrm{s}}\right) \\
& \times \lg K_{L} K_{\mathrm{f}}\left(K_{\rho}-\rho\right)\left(\sigma_{\max }-\sigma_{\mathrm{s} 0}\right)
\end{aligned}
$$

where $K_{\rho}, K_{\mathrm{L}}$, and $K_{\mathrm{f}}$ are the fatigue strength coefficients induced at stress ratio $\rho$, the stress concentration factor induced at cracks in the concrete, and the corrosion pitting factor induced in the corrosion pit in the steel bars, respectively, and $\varepsilon_{\mathrm{c} 0}$ is the initial compressive stress of the steel bars related to the prestress level of the CFRP sheets. The values of the coefficients $K_{\rho}, K_{\mathrm{L}}$, and $K_{\mathrm{f}}$ are detailed in the following paragraphs.

The fatigue strength coefficient $K_{\rho}$ can be determined by (Song 2006):

$$
K_{\rho}=\frac{0.938}{1-0.6165 \rho}
$$

The test data of an RC beam loaded in four-point bending (Heffernan 1997) showed that the localized stresses in the tensile steel reinforcement at the concrete cracks deviated significantly from the nominal stress; These values were consistently $20-40 \%$ higher than the average stress (Hefferan et al. 2004). Deng et al. (2007) tested RC beams strengthened with prestressed aramid fiber-reinforced polymer (AFRP) sheets and found that the prestressed AFRP sheets significantly affected the cracking and stress range of steel reinforcement. A regression analysis was performed based on the experimental test results reported by Heffernan and Deng, and the form of the equation is given as follows:

$$
K_{\mathrm{L}}= \begin{cases}1.30-\alpha_{\mathrm{p}} & \left(0 \leq \alpha_{\mathrm{p}} \leq 0.24, M_{\text {max }}^{\mathrm{f}}>M_{0}\right) \\ 1.05 & \left(0.24 \leq \alpha_{\mathrm{p}}, M_{\max }^{\mathrm{f}}>M_{0}\right)\end{cases}
$$

where $M_{\max }^{\mathrm{f}}$ is the maximum fatigue flexural moment, $M_{0}$ is the decompression flexural moment of the strengthening beams, and $\alpha_{\mathrm{p}}$ is the effective partial prestressing ratio (PPR), which is defined as:

$$
\operatorname{PPR}=\alpha_{\mathrm{p}}=\frac{\left(M_{\mathrm{u}}\right)_{\mathrm{p}}}{\left(M_{\mathrm{u}}\right)_{\mathrm{p}+\mathrm{s}}}=\frac{A_{\mathrm{f}} f_{\mathrm{fu}}^{0}}{A_{\mathrm{f}} f_{\mathrm{fu}}^{0}+A_{\mathrm{sc}} f_{\mathrm{yc}}}
$$

where $\left(M_{\mathrm{u}}\right)_{\mathrm{p}}$ and $\left(M_{\mathrm{u}}\right)_{\mathrm{p}+\mathrm{s}}$ are the ultimate moment of the prestressed CFRP sheets and the total ultimate moment, respectively, $A_{\mathrm{f}}$ is the area of the CFRP sheet, and $f_{\mathrm{fu}}^{0}$ is the ultimate tensile strength of the CFRP composite.
As mentioned previously, pitting corrosion produces a localized reduction in the steel cross-sectional area, but accurately measuring the pit configuration, depth, and distribution is difficult. Therefore, a large deviation exists between how the degree of corrosion and the reduction in steel area are defined based on the corrosion pit depth. Thus, the degree of corrosion in rebar $\left(\eta_{\mathrm{s}}\right)$ is quantified based on the average mass loss. In Eq. (6), the crosssectional area of the corroded steel rebar $A_{\mathrm{sc}}$ is also the average cross-sectional area of the rebar. The average mass loss does not account for the influence of local corrosion pitting on the rebar mechanical properties. The test data of RC beams showed that the localized stresses at the corrosion pits were greater than the theoretical average stresses (Ai-Hammoud et al. 2011). For the fatigue analysis of corroded steel reinforcement, the influence of a notch on the stress range should be reflected in factor $K_{\mathrm{f}}$. Due to the scarcity of published data on the effect of the corrosion notch depth on the range of steel stresses in CFRP-strengthened RC beams under fatigue loading, a linear regression analysis was performed based on the experimental test results reported by Ai-Hammoud et al. (2011). The form of the equation is given as follows $\left(R^{2}=0.942\right)$ :

$$
K_{\mathrm{f}}=0.212 \lambda+1.0
$$

where $\lambda$ is the maximum pit depth.

The relationship between the maximum cross-sectional corrosion degree $\eta_{\mathrm{s}, \text { amx }}$ and the average mass loss $\eta_{\mathrm{s}}$ was calculated as shown in reference (An et al. 2005):

$$
\eta_{\mathrm{s}, \mathrm{amx}}=0.0345+1.2561 \eta_{\mathrm{s}} \quad\left(\eta_{\mathrm{s}} \leq 50 \%\right)
$$

Next, the corrosion pitting factor $K_{\mathrm{f}}$ can be derived from Eqs. (14) and (15):

$$
K_{\mathrm{f}}=1.0+3.39\left(1-\sqrt{1.0-1.25 \eta_{\mathrm{s}}}\right)
$$

Corrosion pits provide sites for fatigue crack initiation, and fatigue crack evolution under repeated loading further reduces the cross-sectional area of rebar. As the fatigue cracks grow, the effective stress reaches the yield stress at some locations. The bar is assumed to abruptly break at failure when the localized fatigue stress $\sigma_{\mathrm{s}, \max }^{\mathrm{f}}$ reaches the yield stress, as shown in Eq. (17):

$$
\sigma_{\mathrm{s}, \max }^{\mathrm{f}} \geq f_{\mathrm{yc}}
$$

where $\sigma_{\mathrm{s}, \max }^{\mathrm{f}}$ is the rebar stress corresponding to the maximum fatigue load at $n$ cycles of repeated loading.

\subsubsection{Carbon Fiber-Reinforced Polymers}

For a fiber-reinforced polymer (FRP) composite, fatigue loading decreases the modulus $E_{\mathrm{f}}$, as observed by Bigaud and Ali (2014) and Ferrier et al. (2011). Based on these studies, the deterioration coefficient $D_{E, f}$ of a prestressed CFRP composite can be expressed as: 


$$
D_{E, f}=1-0.051 \log n
$$

The ultimate tensile strain $\varepsilon_{f, \text { ult }}$ is assumed to be constant over time, and the modulus $E_{\mathrm{f}}^{\mathrm{f}}$ and residual strength $f_{\mathrm{fr}}$ decrease as the number of cycles increases according to the expression:

$$
\begin{aligned}
& E_{f}^{f}(n)=E_{f}^{0} \times D_{E, f} \\
& f_{f r}(n)=f_{f u}^{0} \times D_{E, f}
\end{aligned}
$$

where $E_{\mathrm{f}}^{0}$ and $f_{\mathrm{fu}}^{0}$ are the initial modulus and the ultimate tensile strength of the CFRP composite, respectively.

The fatigue failure process indicates that CFRP sheets fail when the maximum fatigue stress $\sigma_{\mathrm{f} \text { max }}^{\mathrm{f}}$ reaches the residual strength of the CFRP $f_{\text {fr }}$; thus, the residual strength provides a safe fatigue failure criterion, which is defined by the following equation:

$$
\sigma_{\mathrm{f}, \max }^{\mathrm{f}} \geq f_{\mathrm{fr}}(n)
$$

\subsection{Fatigue Bond Properties}

The classical FPM for RC beams strengthened with CFRP sheets assumes a perfect bond between the tension reinforcement and the surrounding concrete. However, previous fatigue experiments showed considerable non-linearity in the strain variation across the beam section under fatigue loading because slippage of the steel reinforcement is likely to occur during fatigue loading. Therefore, it is difficult for the classical FPM to accurately predict the strain and stress distributions across the beam section. Oudah and El-Hacha (2013a, b) developed an FPM that considers the effect of fatigue loading on the degradation of the tension reinforcement bond. The compatibility equations of RC beams strengthened with CFRP sheets is described as follows:

$$
\begin{aligned}
& \varepsilon_{\mathrm{s}}=\left(\frac{h_{0}-x_{\mathrm{n}}}{x_{\mathrm{n}}}\right) \varepsilon_{\mathrm{c}} \gamma_{\mathrm{s}} \\
& \varepsilon_{\mathrm{f}}=\left(\frac{h-x_{\mathrm{n}}}{x_{\mathrm{n}}}\right) \varepsilon_{\mathrm{c}} \gamma_{\mathrm{f}}
\end{aligned}
$$

where $\varepsilon_{\mathrm{S}}$ is the strain in the tensioned steel at the cracked section, $\varepsilon_{\mathrm{f}}$ is the strain in the CFRP, $\varepsilon_{\mathrm{c}}$ is the strain in the concrete at its top fiber, $h_{0}$ is the depth from the top fiber to the level of the tensioned steel, $h$ is the depth from the top fiber to the CFRP level or the beam height, $x_{\mathrm{n}}$ is the depth from the top fiber to the neutral axis, $\gamma_{s}$ is the strain compatibility factor for the steel, and $\gamma_{\mathrm{f}}$ is the strain compatibility factor for the CFRP. The values of $\gamma_{s}$ and $\gamma_{f}$ depend on the bond properties of the steel reinforcement and the CFRP sheets, respectively. The average value of $\gamma_{f}$ is approximately 0.88 (Song and Yu 2015). The average value of $\gamma_{\mathrm{s}}$ for the corroded beam is selected based on a regression analysis of the experimental data (Song and Yu 2015):

$$
\gamma_{\mathrm{s}}=-1.1257 \eta_{\mathrm{s}}+0.9124 \quad\left(\eta_{\mathrm{s}} \leq 0.30\right)
$$

\subsection{Cross-Sectional Stress Analysis of the Beam}

The development of the FAM for corroded RC beams strengthened by prestressed CFRP sheets depends on the following basic assumptions:

- The compressive strain variation of the concrete zone is linear from the neutral axis to the outer fiber, and the strains and stresses follow Hooke's law.

- The tensile resistance of the concrete is neglected.

- The thickness of the CFRP sheets is neglected when the resisting moment is calculated.

- Premature delamination failure of CFRP sheets is not considered because the anchorage prevents such a failure from occurring.

- To simulate the fatigue behavior of the CFRP-strengthened corroded beams, the analysis is conducted in increments of cycles, and the concrete, steel, and CFRP fatigue properties are updated at the end of each cycle increment.

Based on these assumptions, strains that exist in a strengthened corroded beam subjected to a fatigue flexural moment $M^{\mathrm{f}}$ can be modeled as shown in Fig. 1. The beam has a width of $b$, an effective depth of $h_{0}$, a steel strain of $\varepsilon_{\mathrm{s}}$, a concrete strain of $\varepsilon_{\mathrm{c}}$, a total CFRP sheet strain and an initial CFRP sheet strain of $\varepsilon_{\mathrm{f} 0}$; this beam is reinforced by steel rebar with a cross-sectional area of $A_{\mathrm{s}}$ and is strengthened by a CFRP sheet with an area of $A_{\mathrm{f}}$.

Based on the above assumptions and Eqs. (22)-(24), the compatibility equations of the strengthened beams are stated as follows:

$$
\begin{gathered}
\frac{\gamma_{\mathrm{sc}} \varepsilon_{\mathrm{c}}}{x_{\mathrm{n}}}=\frac{\varepsilon_{\mathrm{s}}}{h_{0}-x_{\mathrm{n}}} \\
\frac{\gamma_{\mathrm{f}} \varepsilon_{\mathrm{c}}}{x_{\mathrm{n}}}=\frac{\varepsilon_{\mathrm{f}}-\varepsilon_{\mathrm{f} 0}}{h-x_{\mathrm{n}}}
\end{gathered}
$$

where $\varepsilon_{\mathrm{f}}$ and $\varepsilon_{\mathrm{f} 0}$ are the total strain and the initial effective strain related to the prestress level of the CFRP sheets, respectively.

Equating the tensile and compressive force yields the following relationship:

$$
\frac{1}{2} E_{\mathrm{b}}^{\mathrm{f}} \varepsilon_{\mathrm{c}} b x_{\mathrm{n}}=A_{\mathrm{s}}^{\mathrm{f}} E_{\mathrm{s}} \varepsilon_{\mathrm{s}}+A_{\mathrm{f}} E_{\mathrm{f}}^{\mathrm{f}} \varepsilon_{\mathrm{f}}
$$

where $E_{\mathrm{b}}^{\mathrm{f}}$ is the fatigue deformation modulus of concrete under repeated loading $\left(E_{\mathrm{b}}^{\mathrm{f}}=0.76 E_{\mathrm{c}}^{\mathrm{f}}\right)$.

Equating the applied moment and the internal forces yields the following relationship:

$$
M^{\mathrm{f}}=A_{\mathrm{s}}^{\mathrm{f}} E_{\mathrm{s}} \varepsilon_{\mathrm{s}}\left(h_{0}-\frac{x_{\mathrm{n}}}{3}\right)+A_{\mathrm{f}} E_{\mathrm{f}}^{\mathrm{f}} \varepsilon_{\mathrm{f}}\left(h-\frac{x_{\mathrm{n}}}{3}\right)
$$

\subsection{Fatigue Analysis Flowchart for the Beam}

The above model was implemented in the fatigue analysis of the strengthened corroded beams following the steps listed in Fig. 2. Additional details are provided below: 


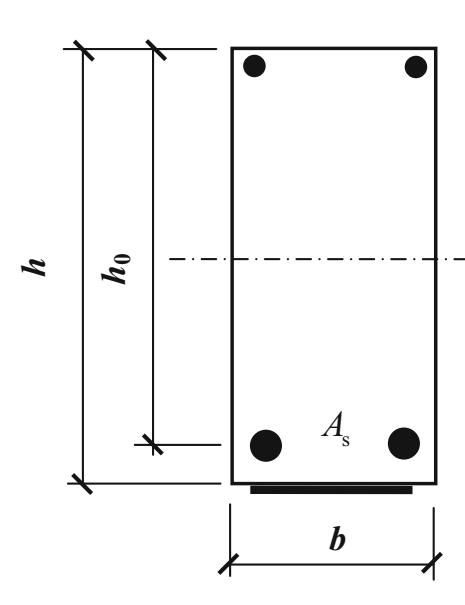

Cross section

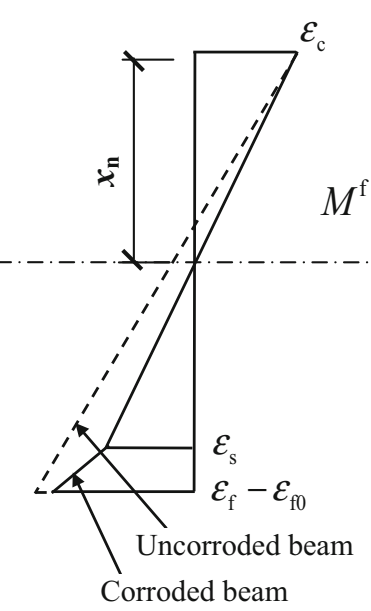

Strain

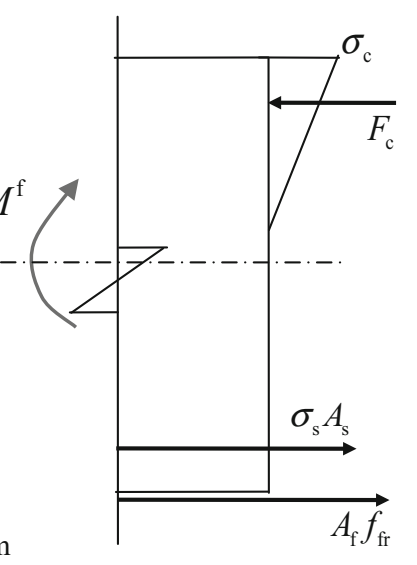

Internal forces

Fig. 1 Stress and strain distributions in beam cross-section.

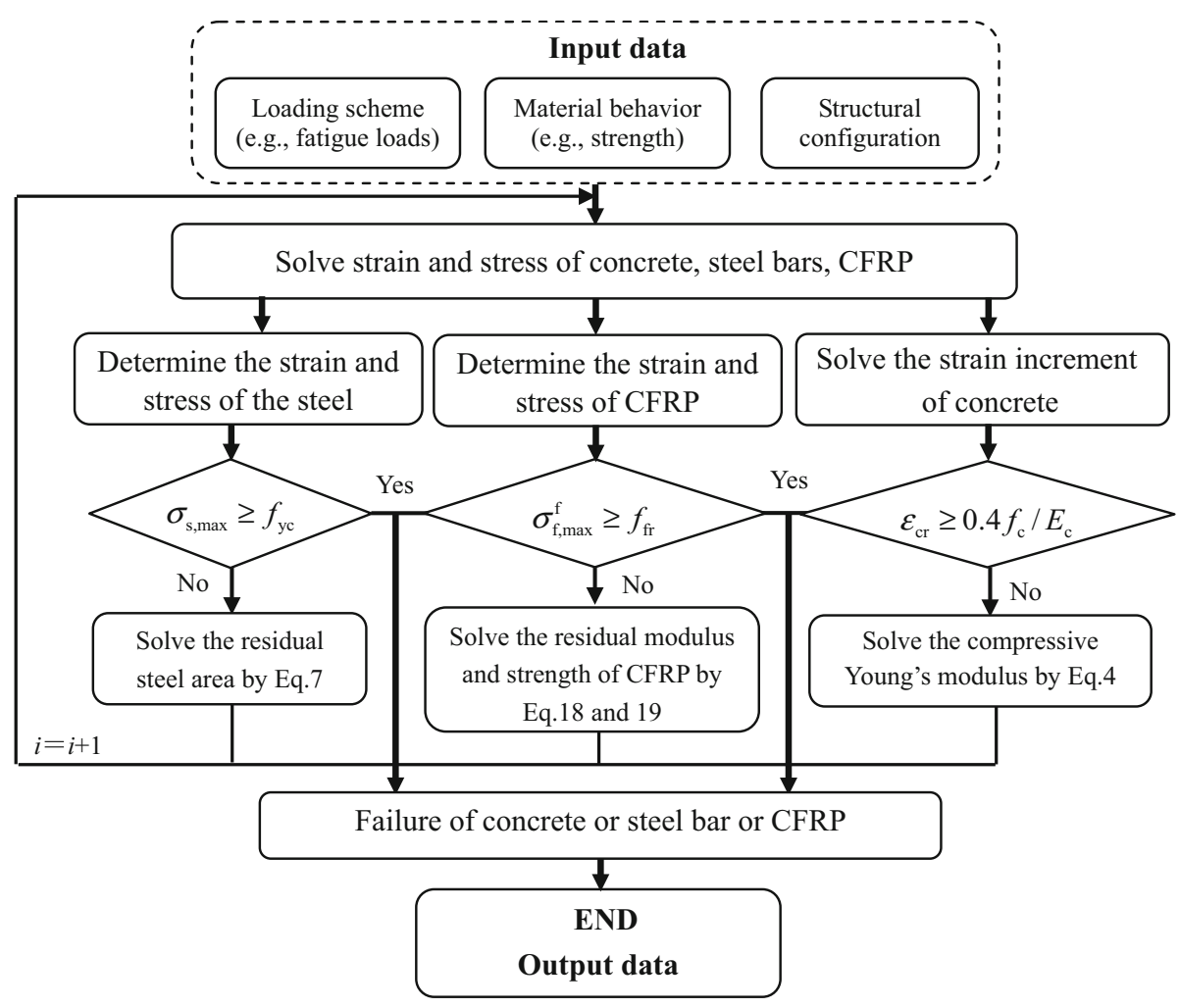

Fig. 2 Fatigue analysis flowchart for the prestressed strengthened corroded beam.

(1) Input the loading scheme, initial material parameters, and specimen configuration, including the fatigue load $\left(M^{\mathrm{f}}\right)$, the initial material parameters $\left(A_{\mathrm{s}}, E_{\mathrm{s}}, f_{\mathrm{y} 0}, \eta_{\mathrm{s}}, E_{\mathrm{c}}\right.$, $f_{\mathrm{c}}, A_{\mathrm{f}}, E_{\mathrm{f}}, f_{\mathrm{f}}$, and $\left.\varepsilon_{\mathrm{fo}}\right)$, and the specimen configuration $\left(b, h\right.$, and $\left.h_{0}\right)$.

(2) Determine the stress and strain in the concrete, rebar, and CFRP sheets at the end of each group of cycles during the fatigue life of the structure using Eqs. (25) (28).

(3) Assess the fatigue states of the concrete, rebar, and CFRP sheets using Eqs. (5), (17), and (21), respectively.

(4) In the subsequent fatigue cycles, update the material properties, including the deformation modulus of the concrete $\left(E_{\mathrm{c}}^{\mathrm{f}}\right)$, the residual cross-sectional area of the corroded steel $\left(A_{\mathrm{s}}^{\mathrm{f}}\right)$, and the elastic modulus of the CFRP sheet ( $\left.E_{\mathrm{f}}^{\mathrm{f}}\right)$ using Eqs. (4), (7), and (19), respectively. Then, return to step (2).

(5) End the program, and output the gathered information.

\subsection{Validation of the Proposed Analysis Model}

The available literature provides only limited test information on the fatigue performance of corroded beams strengthened with prestressed CFRP; additionally, most published studies have presented an insufficient quantity of test results. The proposed FAM is validated by the experimental results of two uncorroded beams that were strengthened with prestressed CFRP sheets and were tested 
by $\mathrm{He}$ et al. (2011). The beams had dimensions of $150 \mathrm{~mm} \times 300 \mathrm{~mm} \times 2000 \mathrm{~mm}$ with spans of $1800 \mathrm{~mm}$. The diameter of the tensioned longitudinal rebar was $14 \mathrm{~mm}$, and the average compressive strength and modulus of elasticity of the concrete were $32.5 \mathrm{MPa}$ and $35.6 \mathrm{GPa}$, respectively. The yield strength of the $14 \mathrm{~mm}$ diameter deformed bars was $335 \mathrm{MPa}$. The mechanical properties of the cured CFRP system were tested and showed an ultimate strength of $3208 \mathrm{MPa}$ and a modulus of elasticity of 234 GPa. A single prestressed layer of CFRP sheet that was $140 \mathrm{~mm}$ wide and $0.167 \mathrm{~mm}$ thick was used to strengthen the beam in flexure. The initial prestressing level in the CFRP sheets was 59 and $60 \%$ of the ultimate strength of the CFRP sheets for Beam 1 and Beam 2, respectively. The maximum and minimum flexural moments in the fatigue load cycles were 21.1 and $6.3 \mathrm{kN} \mathrm{m}$ for Beam 1 and 27.3 and $8.2 \mathrm{kN} \mathrm{m}$ for Beam 2, respectively.

Beam 1 experienced two million cycles of loading without failure. Conversely, Beam 2 experienced fatigue failure in its rebar that was accompanied by a sudden extension of the flexural cracks and a sudden increase in deflection, eventually resulting in the partial rupture of the CFRP sheets. The assessment results showed that the two specimens failed via rupture of the steel rebar, which is identical to the failure mode of the tested items. Figure 3 shows the plots of the fatigue life curve for Beam 2 after different prestress losses. The assessment results with $17.7 \%$ prestress loss correlated well with the experimental data. Figure 4 shows the plots of the strain curves after different numbers of fatigue loading cycles for two beams with $17.7 \%$ prestress loss. The figures clearly show that the assessment results correlated well with the experimental data in all stages of loading behavior up to failure. The scatter of the strain values in the beams occurred because the cross section was at the mid-section of the beams in the assessment and the tests even though the fracture cross section in the tests may deviate from the mid-section.

\section{Model Application}

\subsection{Corroded RC Beams}

The fatigue behavior of beams was modeled using the FAM, and the results produced were verified using He's

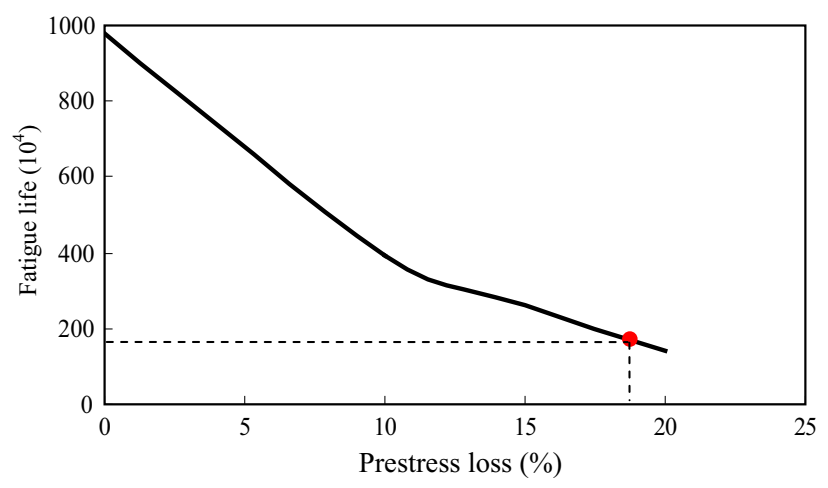

Fig. 3 Fatigue life comparison of experimental and FAM results.

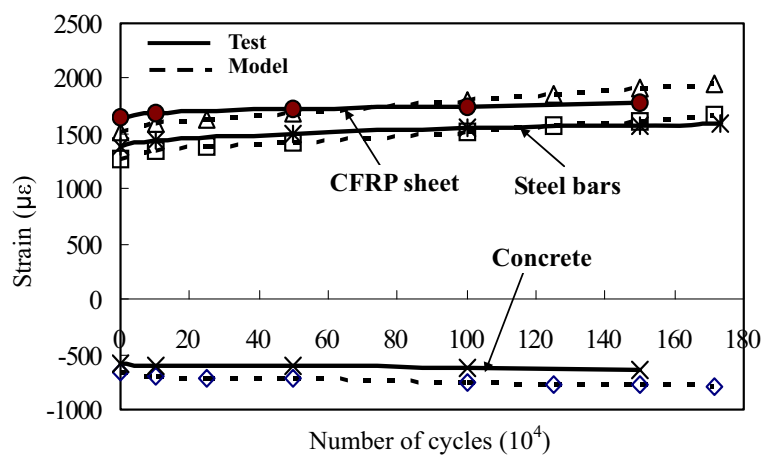

(a)

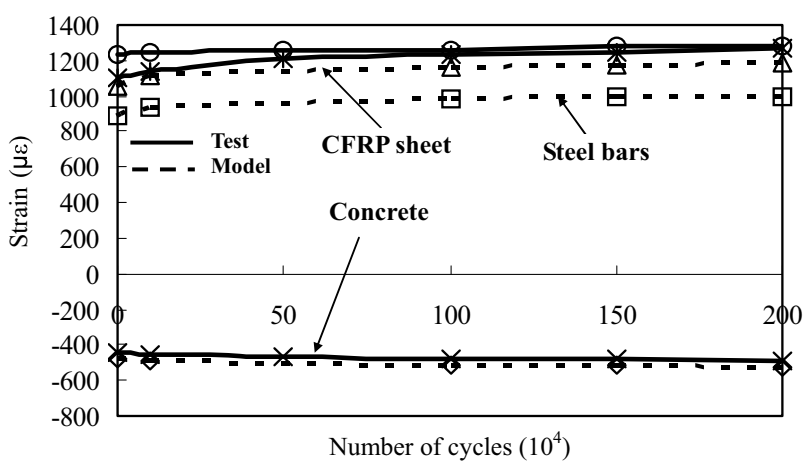

(b)

Fig. 4 Strain comparison of experimental and FAM results: a Beam1, b Beam 2.

experimental data. He's test beams were used to study the effect of the steel corrosion degree, the fatigue load range, and the prestress level on the fatigue life, fatigue strength, stiffness, and post-fatigue ultimate capacity. The test beams were assumed to be corroded at a low level (i.e., $0-5 \%$ average mass loss), a medium level (i.e., 5-10\% average mass loss) and a high level (i.e., 10-20\% average mass loss) and were subsequently strengthened with a single layer of a prestressed CFRP sheet. The ACI Committee 440 (2000) recommends that the maximum prestressed tensile stress of CFRP sheets is limited to 0.55 of their ultimate strength to avoid brittle fracture. Previous studies have also shown that the loss of prestress in CFRP sheets averages $10-30 \%$ of the control tensile stress (Yang and Li 2010). Thus, in the model, the maximum tensile prestress of the CFRP sheets was limited to 0.55 of the ultimate strength, and the effective prestress levels in the CFRP sheets were 0, 2742, and 4113 $\mu \varepsilon$, which were approximately 0,20 , and $30 \%$ of the ultimate strain of the CFRP sheets, respectively. The minimum fatigue flexural moment was determined to be $8.2 \mathrm{kN} \mathrm{m}$, and the maximum fatigue flexural moments were 13.4, 14.9, 16.4, and $17.9 \mathrm{kN} \mathrm{m}$, which represented 45.0, 50.0, 55.0, and $60.0 \%$ of the ultimate strength of the uncorroded, unstrengthened beam, respectively.

\subsection{Fatigue Life}

The RC beam was corroded to different levels and subsequently strengthened with one layer of CFRP flexural sheets. Under cyclic fatigue loading, the unstrengthened and strengthened beams exhibited similar primary fatigue failure 
modes. In all cases, the failure was due to brittle fatigue fracture of the steel reinforcing bars. Figure 5 shows the effect of corrosion and prestressed CFRP strengthening on the beam fatigue life. The vertical arrows at the beginning of the fatigue curve indicate that the specimen did not fail after $200,000,000$ cycles. The fatigue lives of the uncorroded, unstrengthened beams under $0.50,0.55$ and 0.60 of the ultimate strength were 2,176,000 cycles, 1,127,000 cycles and 587,000 cycles, respectively. The beam fatigue life is sensitive to the amount of corrosion present in the steel bars. Using one layer of non-prestressed CFRP sheets to repair the beams with $0-20 \%$ corrosion increased their fatigue life by a factor of 2.35-4.35. In contrast, one layer of non-prestressed CFRP sheets restored the fatigue life of the beam with $4.6 \%$ corrosion to a level nearly equal to that of the uncorroded beam. The strengthening effect was more significant in the beams repaired with prestressed CFRP sheets. Using the

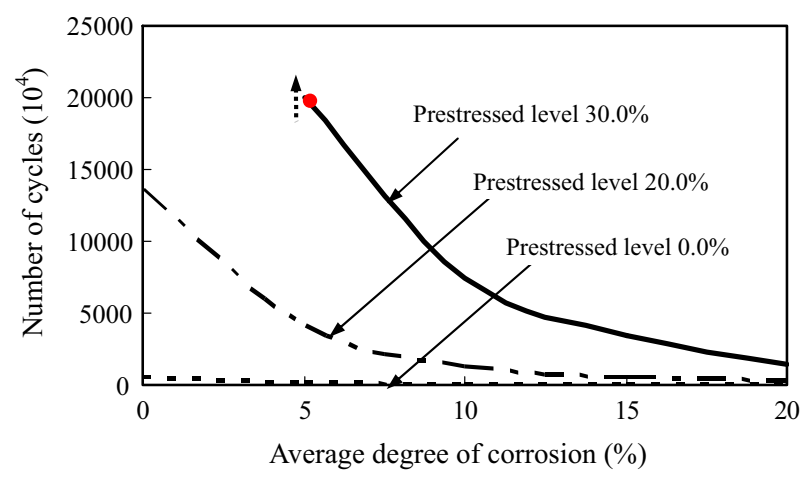

(a)

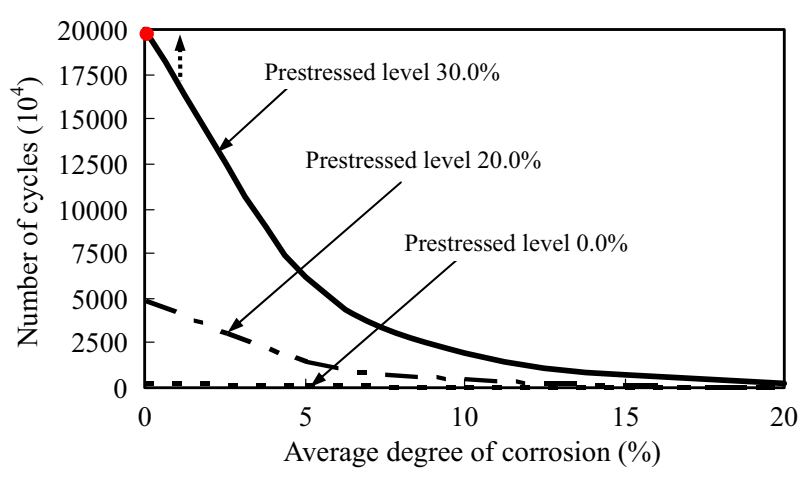

(b)

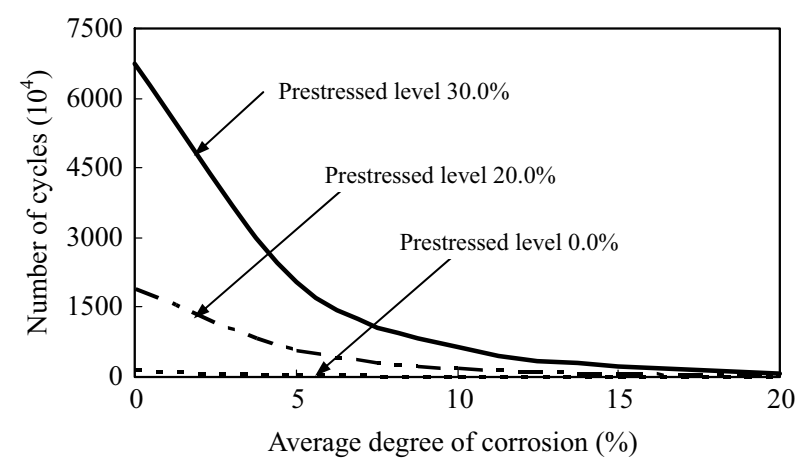

(c)

Fig. 5 Fatigue life versus average degree of corrosion: a 0.5 load level, b 0.55 load level, c 0.60 load level. 20\%-prestressed CFRP sheets to repair the beams with $0-20 \%$ corrosion increased their fatigue life more than 10.0 times on average, and using the $30 \%$-prestressed CFRP sheets to repair the beams with $0-20 \%$ corrosion increased their fatigue life by more than 45.0 times on average. Using the 20- and 30\%-prestressed CFRP sheets to repair the beams with 14.8 and $20.0 \%$ corrosion, respectively, restored their fatigue life to approximately that of the uncorroded beams. These results indicate that corrosion has a significant effect on the fatigue life and that strengthening beams with one layer of non-prestressed CFRP sheets at low corrosion degrees can restore the fatigue life of corroded beams to the level of the uncorroded beams. The strengthening effect was more significant for prestressed CFRP sheets, which can restore the fatigue life of corroded beams with a high corrosion degree to the level of the uncorroded beams.

\section{3 $S-N$ Curves}

The amplitude of cyclic loading significantly affects the fatigue performance, and the $S-N$ relationship was determined for loads that were $0.20,0.25,0.30$, and 0.35 of the uncorroded, unstrengthened beam's ultimate capacity. Figure 6 shows the load-fatigue life curves of the uncorroded beam, the beams strengthened with non-prestressed CFRP, and the beams strengthened with prestressed CFRP. The results indicated that the corrosion degree affects the fatigue life degradation percentage more significantly than the fatigue strength reduction percentage. Using one layer of nonprestressed CFRP sheet restored the fatigue capacity of only the beam with $5 \%$ corrosion to a level approximately equal to that of the uncorroded beam. Using the 20- and 30\%prestressed CFRP sheets to repair the beams with the 15 and $20 \%$ corrosion degrees, respectively, restored the fatigue capacity to the level of the uncorroded beams. These increases can be primarily attributed to the longitudinal prestressed CFRP sheets that stiffened the beams and reduced the stresses and the stress ratio in the steel reinforcing bars as the fatigue failure of these bars led to the failure of the beams.

\subsection{Stiffness Degradation}

The stiffness after each fatigue loading cycle was calculated to provide a quantitative measurement of the stiffness degradation in the beams. The calculation method involved recording the strain in the steel rebar $\varepsilon_{\mathrm{s}}$, the strain at the top fiber of the concrete at the mid-span $\varepsilon_{\mathrm{c}}$, the effective beam depth beam $h_{0}$, and the predicted curvature radius; the stiffness was then calculated from the curvature radius and the fatigue load for each loading cycle. For each beam, the obtained stiffness values were normalized with respect to the initial stiffness of the uncorroded unstrengthened control beam before fatigue loading was applied.

Figure 7 plots the stiffness degradation versus the number of cycles of the beams under 0.55 of the ultimate load level. The results show that steel corrosion in RC beams does not significantly decrease the initial stiffness before fatigue loading but can significantly increase the stiffness degradation rates under fatigue loading. Using one layer of non- 


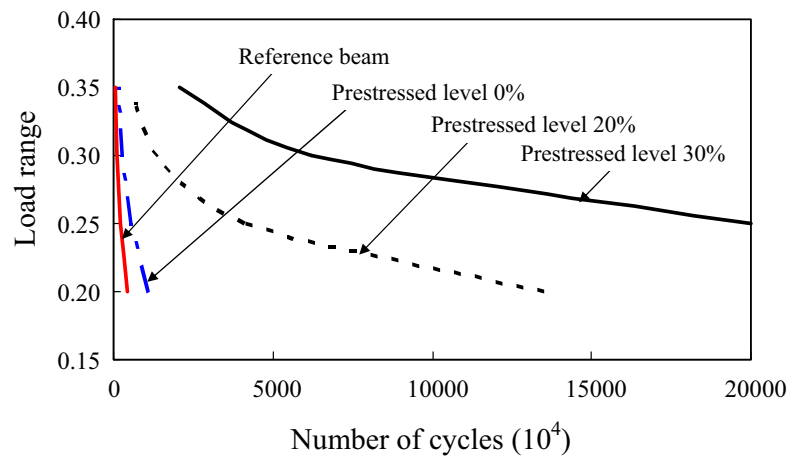

(a)

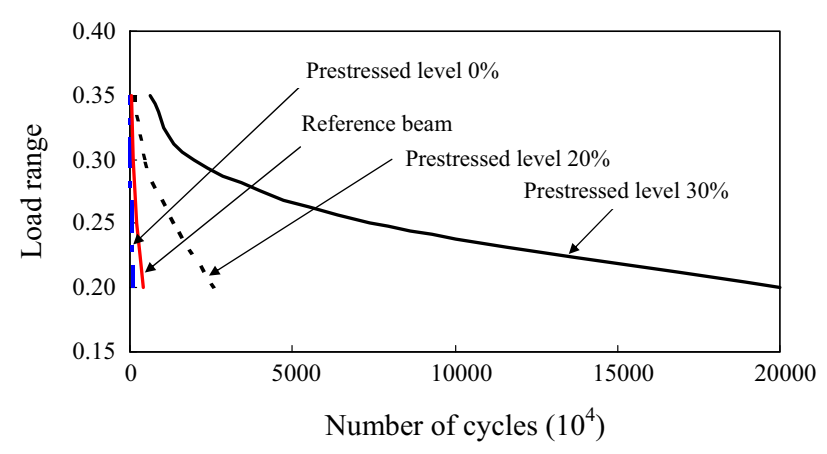

(b)

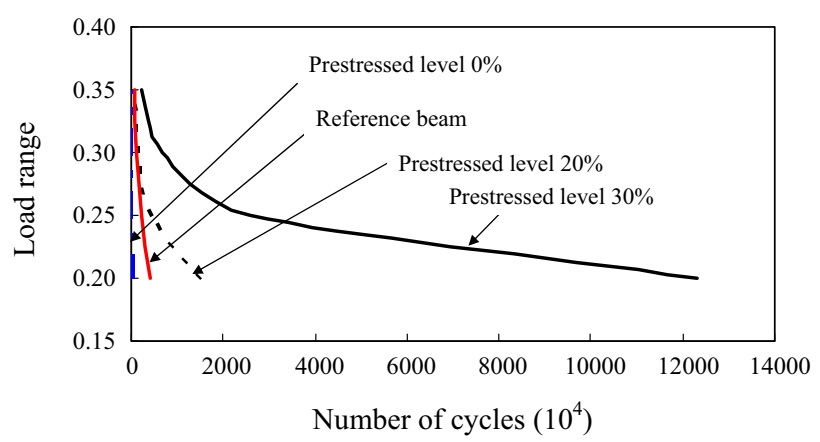

(c)

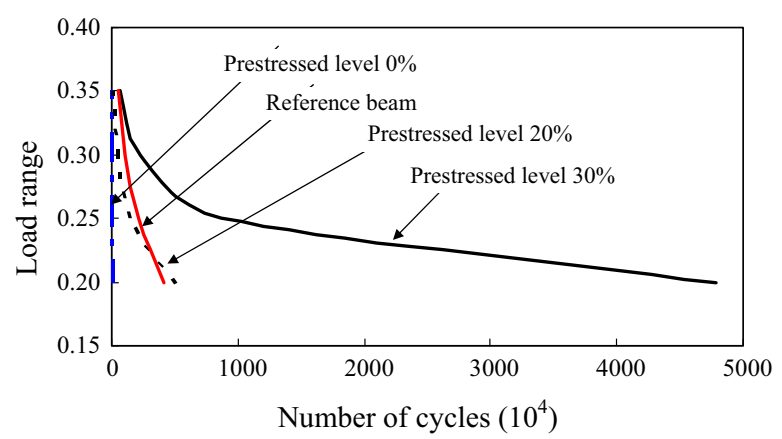

(d)

Fig. 6 Load range versus fatigue life: a 5\% corrosion degree, b $10 \%$ corrosion degree, c $15 \%$ corrosion degree, and d $20 \%$ corrosion degree.

prestressed CFRP sheets restored the fatigue stiffness of only the beam with a low corrosion degree (i.e., $0-4.6 \%$ ) to a level nearly equal to that of the uncorroded beam. Comparing the strengthened and unstrengthened beams, the initial stiffness increased by 26 at $5 \%$ corrosion, 22 at $10 \%$ corrosion, 20 at $15 \%$ corrosion, and 19 at $20 \%$ corrosion for the beams with 20\%-prestressed CFRP flexural sheets; furthermore, the initial stiffness increased by 41 at $5 \%$

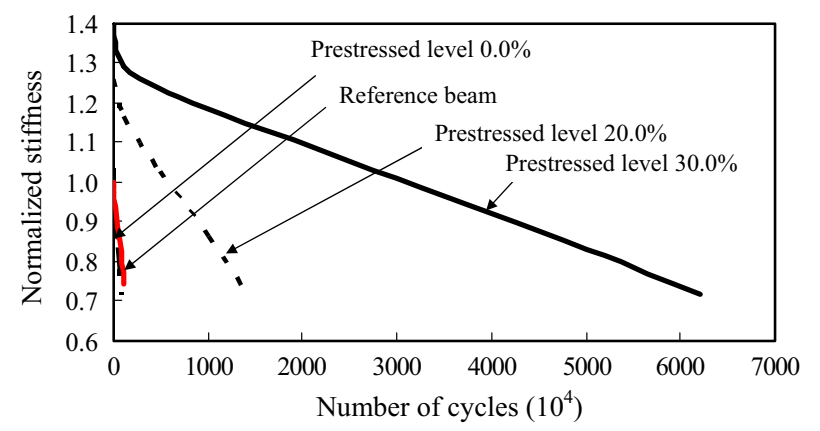

(a)

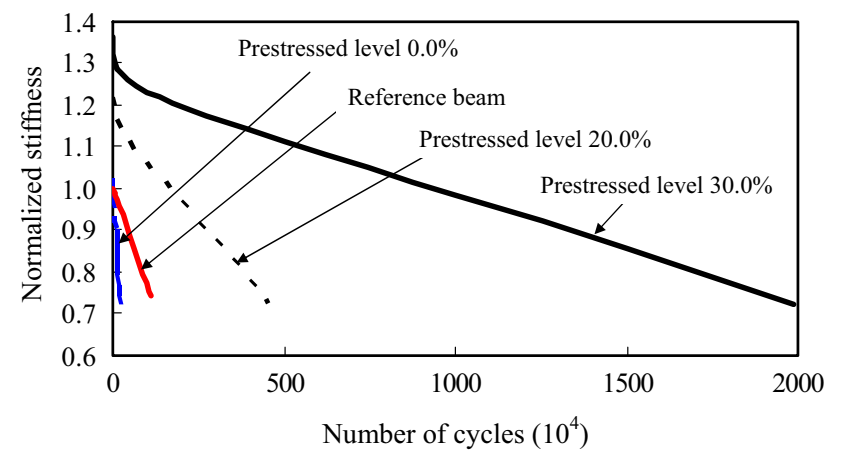

(b)

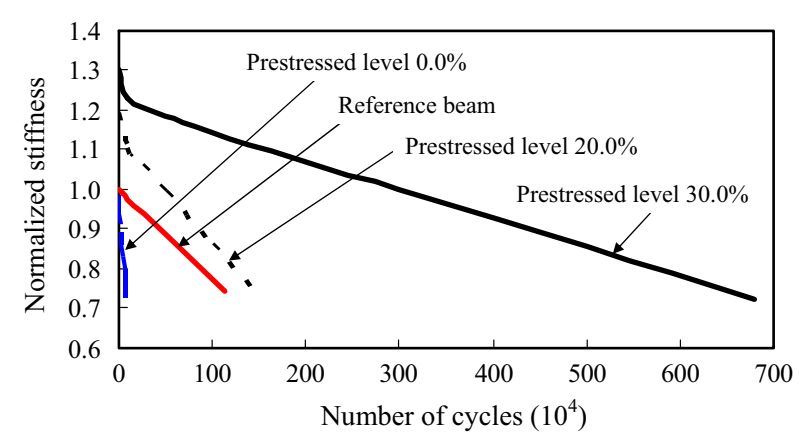

(c)

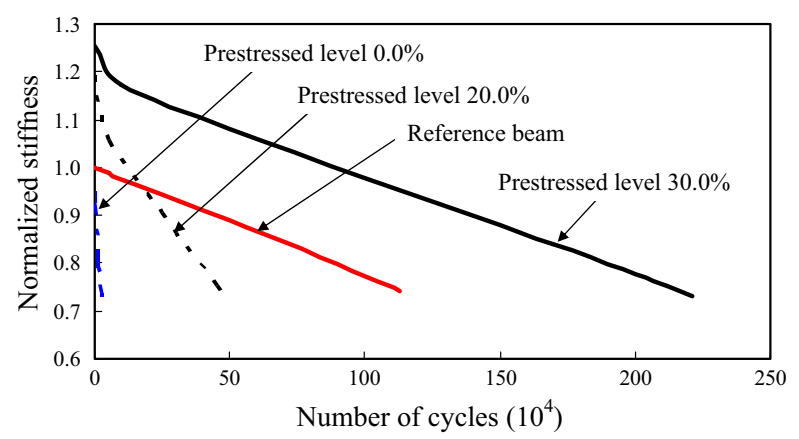

(d)

Fig. 7 Normalized stiffness degradation versus number of cycles: a $5 \%$ corrosion degree, b $10 \%$ corrosion degree, c $15 \%$ corrosion degree, and d $20 \%$ corrosion degree.

corrosion, 36 at $10 \%$ corrosion, 30 at $15 \%$ corrosion, and 25 at $20 \%$ corrosion for the beams with $30 \%$-prestressed CFRP flexural sheets. Comparing the strengthened and unstrengthened beams, the stiffness degradation rates were 0.17 times higher at 5\% corrosion, 0.48 times higher at $10 \%$ corrosion, 1.36 times higher at $15 \%$ corrosion, and 4.16 
times higher at $20 \%$ corrosion for the beams with $20 \%$ prestressed CFRP flexural sheets; furthermore, the stiffness degradation rates were 0.05 times higher at $5 \%$ corrosion, 0.14 times higher at $10 \%$ corrosion, 0.37 times higher at $15 \%$ corrosion, and 1.02 times higher at $20 \%$ corrosion for the beams with 30\%-prestressed CFRP flexural sheets. The results show that repairs with one layer of CFRP sheets that were $0 \%$ (i.e., non-prestressed), 20 , or $30 \%$ prestressed restored the fatigue stiffness of the beams with low $(0-5 \%)$, medium (5-10\%), and high (10-20\%) corrosion degrees to a level nearly equal to that of the uncorroded beams.

Figure 8 shows the stiffness degradation versus the number of cycles for the beams with $15 \%$ corrosion. Comparing the strengthened and unstrengthened beams, the stiffness degradation rates were 0.43 times higher at the $0.45 \mathrm{load}$ level, 0.80 times higher at the 0.50 load level, 1.35 times higher at the 0.55 load level, and 1.97 times higher at the 0.60 load level for the beams with 20\%-prestressed CFRP flexural sheets; furthermore, the stiffness degradation rates were 0.06 times higher at the 0.45 load level, 0.17 times higher at the 0.5 load level, 0.37 times higher at the 0.55 load level, and 0.65 times higher at the 0.60 load level for the beams with 30\%-prestressed CFRP flexural sheets. The stiffness of the beams with the 0.60 load level strengthened with one layer of 20\%-prestressed CFRP sheets after cyclic loading was still less than that of the uncorroded control beam. These results indicate that the load level significantly affected the fatigue stiffness degradation of the beams. The loss of stiffness is exaggerated when corrosion is combined with fatigue loading.

The change in stiffness is related to the opening and propagation of flexural cracks, which implies a relative slip between the concrete, steel reinforcement, and the CFRP sheet as well as fatigue damage of the constituent materials and tensile failure of the concrete. The prestress in the CFRP sheets is the primary reason for the increase in stiffness because prestressed CFRP sheets confine the tensioned face of the beams, reduce the widening of flexural cracks and delay the damage evolution of the steel bars during fatigue cyclic loading.

\subsection{Post-Fatigue Ultimate Strength}

The ultimate strength after each fatigue loading cycle was calculated to provide a quantitative measure of the capacity degradation of the beams. The obtained strength values were normalized with respect to the initial ultimate strength of the uncorroded control beam before fatigue loading was applied, as shown in Fig. 9. Compared to the uncorroded RC beam, the initial capacity of the corroded beams with $10.0 \%$ corrosion at the 0.55 load level increased by $52.0,56$ and $57.0 \%$ with the 0-, 20-, and 30\%-prestressed CFRP sheets, respectively; however, the strength degradation rates of the beams with one layer of non-prestressed CFRP flexural sheets were still less than that of the uncorroded control beam. The beams with $15.0 \%$ corrosion at the $0.60 \mathrm{load}$ level showed similar trends. The beams with a low corrosion degree that were strengthened with one layer of non-prestressed CFRP sheets restored the strength degradation rate

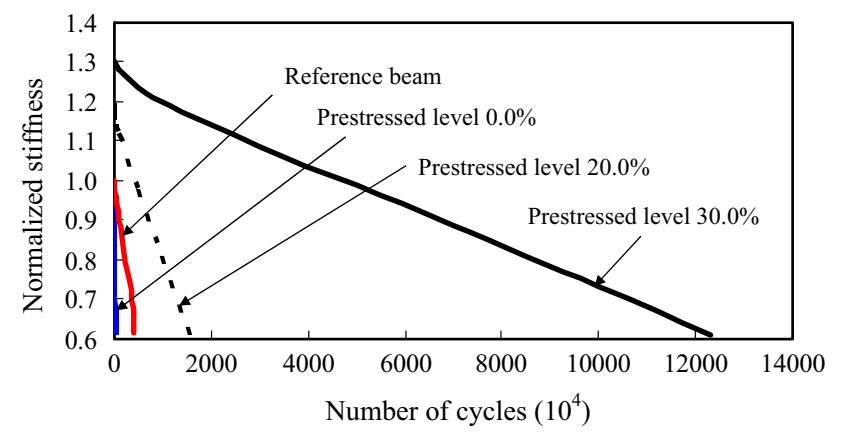

(a)

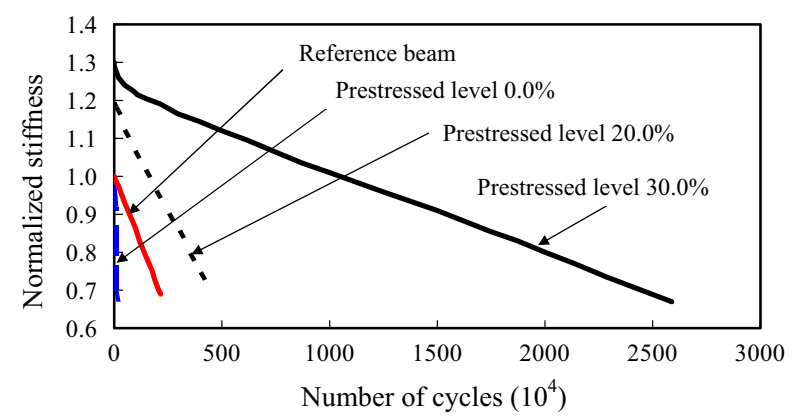

(b)

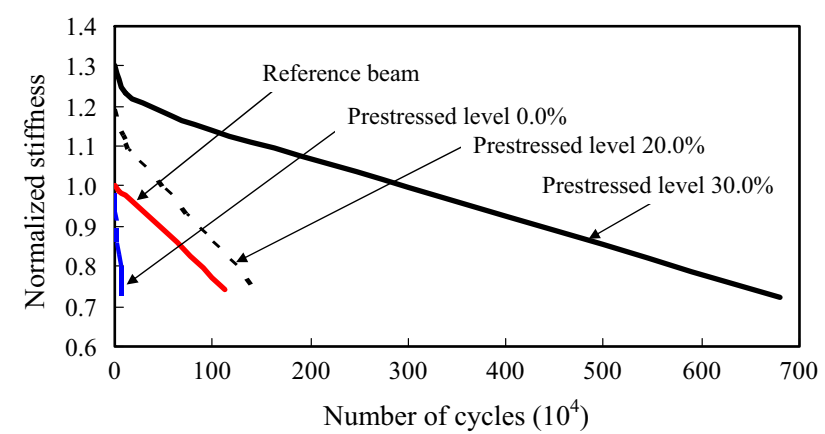

(c)

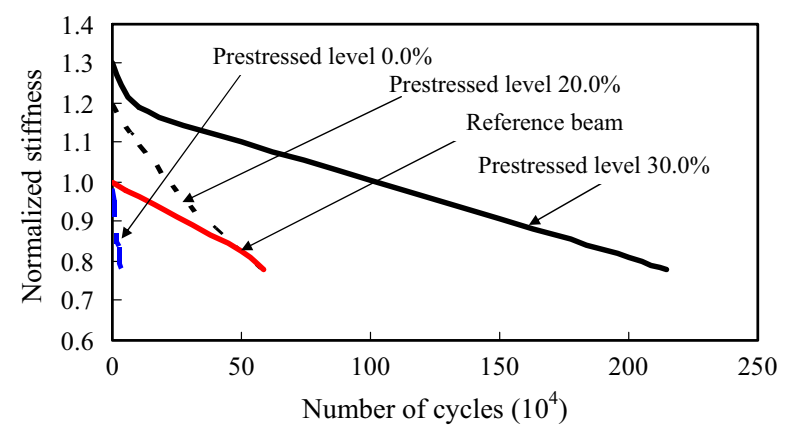

(d)

Fig. 8 Normalized stiffness degradation versus number of cycles: a 0.45 load level, b 0.5 load level, c 0.55 load level, and d 0.60 load level.

to a level approximately equal to that of the uncorroded beams. Using the 20- and 30\%-prestressed CFRP sheets to repair the beams with 15 and $20 \%$ corrosion, respectively, restored the ultimate strength to that of the uncorroded beams. The initial capacity of the beams with prestressed CFRP sheets was more than $4-5 \%$ greater that of the beams with non-prestressed CFRP sheets, which could be attributed 


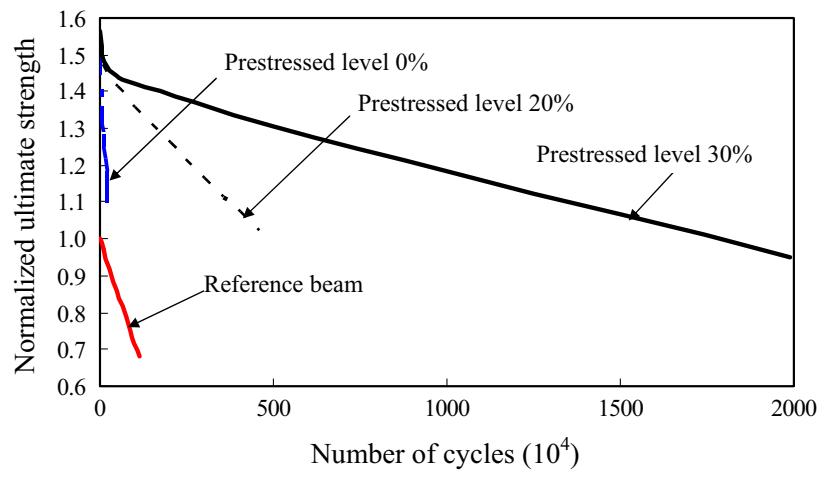

(a)

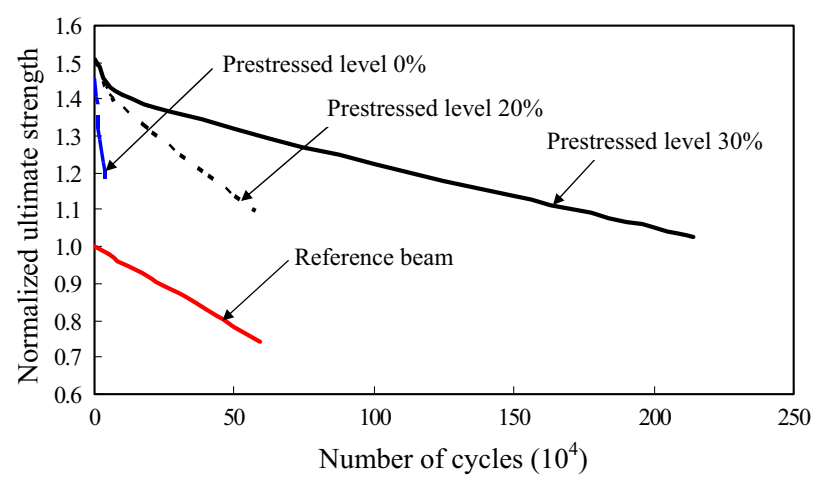

(b)

Fig. 9 Normalized ultimate strength degradation versus number of cycles: a $10 \%$ corrosion degree and 0.55 load level, b $15 \%$ corrosion degree and 0.60 load level.

to the compressive prestress action in the beams provided by the prestressed CFRP sheets. These results indicated that the steel bar corrosion reduced the initial capacity and significantly increased the ultimate strength degradation rates of the structure; the prestressed CFRP sheets were shown to effectively restore the ultimate strength of the corroded beams to that of the uncorroded beams.

\section{Conclusions}

An FAM based on the fatigue properties of the constituent materials and a cross-sectional stress analysis was proposed to assess the fatigue behavior of corroded beams strengthened with prestressed CFRP sheets. Based on the analysis results, the following conclusions can be drawn:

(1) The controlling factor for the fatigue behavior of the beam is the fatigue behavior of the corroded steel bars. The failures of the unstrengthened beams, the beams strengthened with non-prestressed CFRP sheets and the beams strengthened with prestressed CFRP sheets were caused by rupturing of the steel reinforcement.

(2) Using one layer of non-prestressed CFRP sheets to repair beams with rebar at low corrosion degrees increased the beam fatigue life and strength to approximately the values of the uncorroded beams. Using prestressed CFRP sheets restored the beam fatigue life and strength with rebar at medium and high corrosion degrees to the values of the uncorroded beams.

(3) Steel rebar corrosion was shown to reduce the initial stiffness and ultimate strength and to significantly increase the stiffness degradation rates and the ultimate strength degradation rates of the structure. Using one layer of non-prestressed CFRP sheets increased the initial stiffness and strength and decreased the stiffness degradation rates and the strength degradation rates; however, the stiffness and strength of the CFRPstrengthened beams after the loading cycles were still less than the stiffness and strength of the uncorroded control beam. Strengthening with $20-$ and $30 \%$ prestressed CFRP sheets restored the stiffness and strength of the beams with rebar at medium and high corrosion degrees, respectively, to the levels of the uncorroded beams.

(4) Similar to strengthening with non-prestressed CFRP sheets, the factors for the design of prestressed CFRP strengthening of corroded $\mathrm{RC}$ beams fall in the order of stiffness, fatigue life, fatigue strength, and ultimate capacity.

\section{Open Access}

This article is distributed under the terms of the Creative Commons Attribution 4.0 International License (http://creativecommons.org/licenses/by/4.0/), which permits unrestricted use, distribution, and reproduction in any medium, provided you give appropriate credit to the original author(s) and the source, provide a link to the Creative Commons license, and indicate if changes were made.

\section{References}

ACI Committee 440 (2000). Guide for the design and construction of externally bonded FRP systems for strengthening concrete structures, ACI.

Ai-Hammoud, R., Soudki, K., \& Topper, T. H. (2010). Bond analysis of corroded reinforced concrete beams under monotonic and fatigue loads. Journal Cement Concrete Composites, 32(3), 194-203.

Ai-Hammoud, R., Soudki, K., \& Topper, T. H. (2011). Fatigue Flexural behaviour of corroded reinforced concrete beams repaired with CFRP sheets. ASCE Journal Composites for Construction, 15(1), 42-51.

Almusallam, A. A. (2001). Effect of corrosion on the properties of reinforcing steel bars. Journal Construction and Building Materials, 15(8), 361-368.

Al-Rousan, R., \& Issa, M. (2011). Fatigue performance of reinforced concrete beams strengthened with CFRP sheets. Journal Construction and Building Materials, 25(8), 3520-3529. 
An, L., Ouyang, P., \& Zheng, Y. M. (2005). Effect of stress concentration on mechanical properties of corroded reinforcing steel bars. Journal Southeast University, 35(6), 940-944. (in Chinese).

Bastidas-Arteaga, E., Bressolette, P., Chateauneuf, A., \& Sanchez-Silva, M. (2009). Probabilistic lifetime assessment of $\mathrm{RC}$ structures under coupled corrosion-fatigue deterioration processes. Journal Structual Safety, 31(1), 84-96.

Bigaud, D., \& Ali, O. (2014). Time-variant flexural reliability of RC beams with externally bonded CFRP under combined fatigue-corrosion actions. Journal Engineering and System Safety, 131, 257-270.

Correia, L., Teixeira, T., Michels, J., et al. (2015). Flexural behaviour of RC slabs strengthened with prestressed CFRP strips using different anchorage systems. Journal Composites Part B, 81, 158-170.

Deng, Z. C., Zhang, P. F., Li, J. H., \& He, W. P. (2007). Fatigue and static behaviors of $\mathrm{RC}$ beams strengthened with prestressed AFRP. China Journal Highway and Transport, 20(6), 49-55.

EISafty, A., Graeff, M. K., \& Sam Fallaha, S. (2014). Behavior of laterally damaged prestressed concrete bridge girders repaired with cfrp laminates under static and fatigue loading. International Journal of Concrete Structures and Materials, 8(1), 43-59.

El-Hacha, R., Wight, R. G., \& Green, M. F. (2001). Prestressed fibre-reinforced polymer laminates for strengthening structures. Progress in Structural Engineering and Materials, 3, 111-121.

Elrefai, A., West, J., \& Soudki, K. (2012). Fatigue of reinforced concrete beams strengthened with externally post-tensioned CFRP tendons. Journal Construction and Building Materials, 29(4), 246-256.

Ferrier, E., Bigaud, D., Clement, J. C., \& Hamelin, P. (2011). Fatigue-loading effect on RC beams strengthened with externally bonded FRP. Journal Construction and Building Materials, 25(2), 539-546.

Grelle, S. V., \& Sneed, L. H. (2013). Review of anchorage systems for externally bonded FRP laminates. International Journal of Concrete Structures and Materials, 7(1), 17-33.

He, C. S., Wang, W. W., Yang, W., \& Ye, J. S. (2011). Experimental study on fatigue behavior of reinforced concrete beams strengthened by prestressed CFRP sheets. Journal Southeast University, 41(6), 841-847. (in Chinese).

Heffernan, J. P. (1997). Fatigue behavior of reinforced concrete beams strengthed with CFRP laminates. PhD Thesis, Department of Civil Engineering, Royal Military College of Canada, Kingston, Ontario, Canada.

Hefferan, P. J., Erki, M. A., \& DuQuesnay, D. L. (2004). Stress redistribution in cyclically loaded reinforced concrete beams. ACI Structural Journal, 101(2), 261-268.

Holmen, J. O. (1982). Fatigue of concrete by constant and variable amplitude loading. ACI Special Publication, 75, $71-110$.

Kang, T. H. K., Howell, J., \& Kim, S. (2012). A state -of- the art review on debonding failures of FRP laminates externally adhered to concrete. International Journal of Concrete Structures and Materials, 6(2), 123-134.
Kang, T. H. K., Kim, W., Ha, S. S., \& Choi, D. U. (2014). Hybrid effects of carbon-glass FRP sheets in combination with or without concrete beams. International Journal of Concrete Structures and Materials, 8(1), 27-41.

Kim, Y. J., Shi, C., \& Green, M. F. (2008). Ductility and cracking bevavior of prestressed concrete beams strengthened with prestressed CFRP sheets. ASCE Journal Composites for Construction, 12(3), 274-283.

Ma, Y. F., Xiang, Y. B., Wang, L., Zhang, J. R., \& Liu, Y. M. (2014). Fatigue life prediction for ageing RC beams considering corrosion. Journal Engineering Structurs, 79(11), 211-221.

Masoud, S., Soudki, K., \& Topper, T. (2001). CFRP-strengthened and corroded RC beams under monotonic and fatigue loads. ASCE Journal Composites for Construction, 5(4), 228-236.

Michels, J., Sena-Cruz, J., Czaderski, C., \& Motavalli, M. (2013). Structural strengthening with prestressed CFRP strips with gradient anchorage. ASCE Journal Composites for Construction, 17(5), 651-661.

Motavalli, M., Czaderski, C., \& Pfyl-Lang, K. (2011). Prestresseed CFRP for strengthening of reinforced concrete structures: Recent developments at Empa, Switzerland. ASCE Journal Composites for Construction, 15(2), 194-205.

Oudah, F., \& El-Hacha, R. (2013a). Analytical fatigfue prediction model of RC beams strengthened in flexure using prestressed FRP reinforcement. Journal Engineering Structurs, 46, 173-183.

Oudah, F., \& El-Hacha, R. (2013b). Reseach progress on the fatigue performance of $\mathrm{RC}$ beams strengthened in flexure using fiber reinforced polymers. Journal Composites Part $B, 47,82-95$.

Ouezdou, M. B., Belarbi, A., \& Bae, S. W. (2009). Effective bond length of frp sheets externally bonded to concrete. International Journal of Concrete Structures and Materials, 3(2), 127-131.

Ren, W., Sneed, L. H., Gai, Y., \& Kang, X. (2015). Test results and nonlinear analysis of RC T-beams strengthened by bonded steel plates. International Journal of Concrete Structures and Materials, 10(3), 1-11.

Rosenboom, O., \& Rizkalla, S. (2006). Behavior of prestressed concrete strengthened with various CFRP systems subjected to fatigue loading. ASCE Journal Composites for Construction, 10(6), 492-502.

Song, Y. P. (2006). Fatigue behavior and design principle of concrete structures. Beijing: China machine press.

Song, L., \& Yu, Z. W. (2015). Fatigue performance of corroded reinforced concerete beams strengthened with CFRP sheets. Journal Construction and Building Materials, 29(5), 99-109.

Triantafillou, T. C., Deskovic, N., \& Deuring, M. (1992). Strengthening of concrete structures with prestressed fiber reinforced plastic sheets. ACI Structural Journal, 89(3), 235-244.

Wang, W. W., Dai, J. G., Harries, K. A., \& Bao, Q. H. (2012). Prestress losses and flexural behavior of reinforced concrete beams strengthened with posttensioned CFRP sheets. 
ASCE Journal Composites for Construction, 16(2), 207-216.

Yang, Y. X., \& Li, Q. W. (2010). Technology of strengthening concrete structures with prestressed carbon fiber reinforced polymer sheets. Beijing: China chemical industry press.
Yi, W. J., Kunnath, S. K., Sun, X. D., Shi, C. J., \& Tang, F. J. (2010). Fatigue behavior of reinforced concrete beams with corroded steel reinforcement. ACI Structural Journal, 107(5), 526-533. 